\title{
Cavity formation in split ring resonators
}

\author{
Humeyra Caglayan $^{\mathrm{a}, *}$, Irfan Bulu $^{\mathrm{b}}$, Marko Loncar $^{\mathrm{b}}$, Ekmel Ozbay $^{\mathrm{a}}$ \\ ${ }^{a}$ Nanotechnology Research Center-NANOTAM, Department of Physics, Department of Electrical and Electronics Engineering, \\ Bilkent University, Bilkent 06800, Ankara, Turkey \\ ${ }^{\mathrm{b}}$ School of Engineering and Applied Sciences, Harvard University, 33 Oxford Street, Cambridge, MA 02138, USA
}

Received 22 August 2008; received in revised form 11 September 2008; accepted 11 September 2008

Available online 27 September 2008

\begin{abstract}
We report that it is possible to obtain a cavity structure by the deformation of a unit cell of an split ring resonator (SRR) structure. We presented the Q-factor of the cavity resonance as 192 for an SRR-based single cavity. Subsequently, we brought two and three cavities together with an intercavity distance of two metamaterial unit cells and investigated the transmission spectrum of SRRbased interacting 2-cavity and 3-cavity systems. The splitting of eigenmodes due to the interaction between the localized electromagnetic cavity modes was observed. Eventually, in taking full advantage of the effective medium theory, we modeled SRRbased cavities as 1D Fabry-Perot reflectors (FPRs) with a subwavelength cavity at the center. Finally, we observed that at the cavity resonance, the effective group velocity was reduced by a factor of 67 for an SRR-based single cavity compared to the electromagnetic waves propagating in free space.
\end{abstract}

(C) 2008 Elsevier B.V. All rights reserved.

PACS : 41.20.Jb; 42.70.a

Keywords: Cavity; Split rind resonator; Metamaterials

\section{Introduction}

The magnetic permeability $(\mu)$ of naturally occurring materials is positive. Constructing a negative $\mu$ medium is difficult because of the absence of a magnetic charge. In 1999, Pendry et al. suggested split ring resonator (SRR) structures and demonstrated that such structures strongly respond to an incident magnetic field resulting in negative permeability [1]. An SRR structure is resonant around the magnetic plasma frequency [1-3], which is induced by the currents that imitate magnetic poles.

Split ring resonators have received a great amount of interest and were widely studied for constructing metamaterials. The transmission characteristics of

\footnotetext{
* Corresponding author.

E-mail address: caglay@bilkent.edu.tr (H. Caglayan).
}

periodic [2-10] and disordered SRRs [11] are reported. There are also numerical simulations in order to study the properties of transmission spectra [12-15], effective parameters [16-18], and the magnetic resonances of SRRs [3,19]. Several analytical models are reported for a better understanding of SRRs' resonance behaviors [20-24]. The studies on SRRs and metamaterials are mainly performed in the gigahertz $(\mathrm{GHz})$ frequency regime, but recently magnetic resonances of SRRs at terahertz $(\mathrm{THz})$ frequencies have been obtained both experimentally and theoretically [25,26].

In the present paper, we investigate cavity formation in SRR structures. We introduce the cavity structure and present the transmission of SRR and SRR-based single cavity structures. Our results show that the modification of a unit cell of the metamaterial can exhibit a cavity resonance. Subsequently, we brought two and three cavities together with an intercavity distance of two 


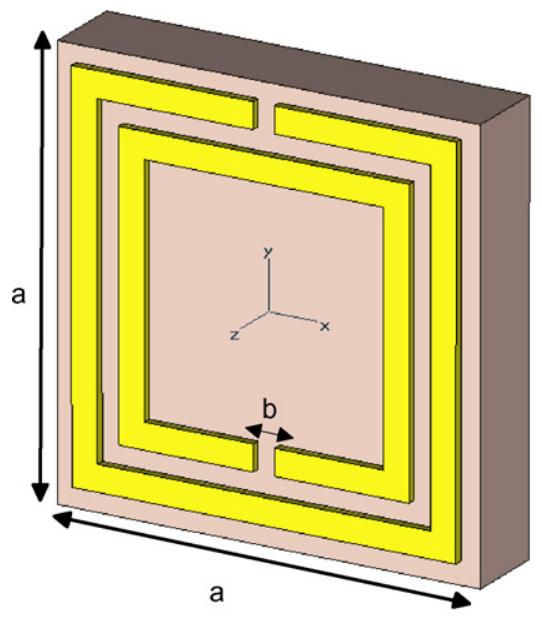

(a)

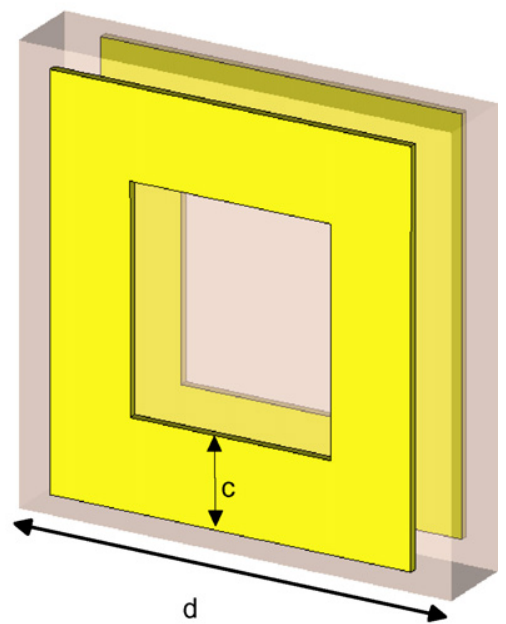

(b)

Fig. 1. (a) The unit cell of the SRR structure: $A=4.95 \mathrm{~mm}, B=0.25 \mathrm{~mm}$. (b) The cavity structure: $C=1 \mathrm{~mm}, D=5.4 \mathrm{~mm}$.

metamaterial unit cells, in which we investigated the transmission spectrum of SRR-based 2-cavity and 3 -cavity systems. Consequently, we modeled SRRbased cavity as a one-dimensional (1D) Fabry-Perot resonator (FPR) with a subwavelength cavity at the center. The transmission was calculated from the FPR model using the 1D transfer matrix method (TMM). Finally, we present here an effective group velocity reduction at the cavity resonance.

\section{Split ring resonator-based single cavity system}

The SRR structure that we used for the present study was a one-dimensional periodic arrangement of square rings. The square SRRs were printed on Teflon $(\varepsilon=2.17)$ substrates. The thickness of the Teflon substrate was $1 \mathrm{~mm}$. The unit cell of the SRR structure is shown in Fig. 1(a). The thickness of the metal (copper) was $0.05 \mathrm{~mm}$. The lattice constant along the $x$ direction was $4.95 \mathrm{~mm}$, and along the $x$ direction (propagation direction) it was $3 \mathrm{~mm}$. There were 40 layers along the $y$ and $z$ directions, and 5 unit cell along the propagation direction. The E-field was in the $y$ direction. The experimental setup consist of an HP $8510 \mathrm{C}$ network analyzer and two standard gain horn antennae in order to measure the transmission amplitude. The simulations throughout the paper were performed by the commercial software program CST Microwave Studio ${ }^{\circledR}$.

The measured transmission demonstrated that the SRR structure has a band gap from 5 to $7 \mathrm{GHz}$ (Fig. 2(a)). This gap is due to the magnetic resonance [27], in which the SRR structure exhibits a negative $\mu$ medium. When the electromagnetic field passes through the ring, an induced current is created, in which the generated field is perpendicular to the magnetic field of
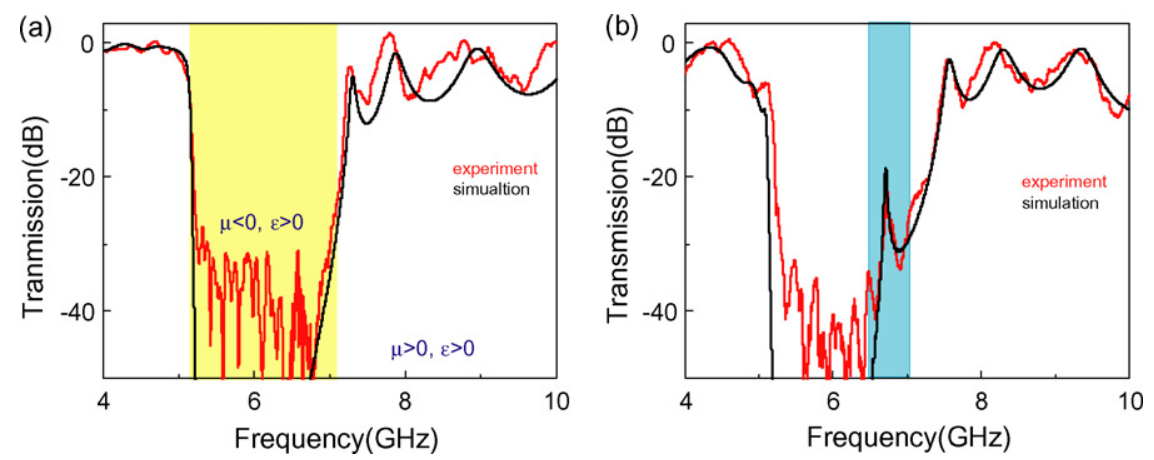

Fig. 2. (a) The SRR structure has a band gap from 5 to $7 \mathrm{GHz}$. (b) A cavity structure is introduced by replacing the center unit cell with a positive index medium. The cavity resonance is observed at $6.7 \mathrm{GHz}(44.7 \mathrm{~mm})$ by the SRR-based single cavity structure. 
the light. The magnetic resonance results in a negative $\mu$. EM waves cannot propagate in the negative $\mu$ medium and possess a band gap in the spectrum. The measured results are in good agreement with the theoretical results.

It is possible to obtain a cavity mode in the forbidden transmission region of the SRR structure by introducing a defect in the structure. The translational symmetry of the negative $\mu$ system can be broken by a positive $\mu$ medium. In the present paper, we changed the center unit cell of the structures by a closed ring structure, which were placed on both sides of the board and possessed positive $\varepsilon$ and $\mu$ (Fig. 1(b)). This deformation in the SRR structures resulted in a cavity mode transmission spectrum. The transmission spectrum of the SRR-based single cavity system is shown in Fig. 2(b). We observed a cavity resonance with the Q-factor (quality factor, defined as the center frequency, divided by the full width at half maximum) of 192 at $\Omega=6.7 \mathrm{GHz}(44.7 \mathrm{~mm}$ ) by the SRR single cavity system.

Moreover, we calculated the transmissions for an SRR cavity structure without loss, with loss on board only, and loss on metal only. The results showed that the transmission at the cavity resonance is decreased because of the loss of the board and metal $(\mathrm{Cu})$ (Fig. 3). The Q-factor for the SRR cavity resonance without a loss is 3290 . On the other hand, the Q-factors are 1100 and 475 for the systems with loss on board only and with loss on metal only, respectively. Therefore, one can obtain metamaterial cavities with higher Q-factors by using different designs and materials.

\section{2-Cavity and 3-cavity systems}

Subsequently, we investigated SRR-based 2-cavity and 3-cavity systems. We brought two and three cavities

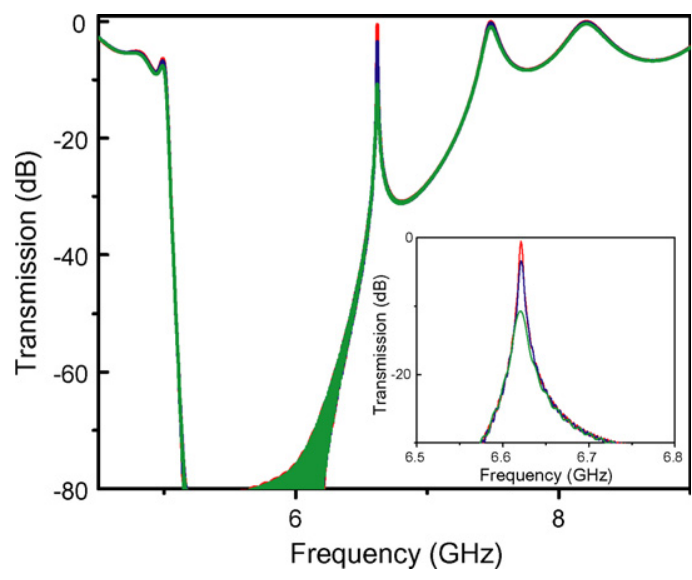

Fig. 3. The calculated transmission for SRR cavity structure without loss (red line), with loss on board only (blue line), and with loss on metal only (green line). The calculated loss free transmission of the SRR cavity resonance possesses the highest Q-factor. It decreases if the losses of the materials have been introduced (inset: zoomed to the cavity resonance frequency). (For interpretation of the references to color in this figure legend, the reader is referred to the web version of the article.)

together with an intercavity distance of two metamaterial unit cells. The total two-cavity system contains two cavities and two metamaterial unit cells on each side of each cavity ( 2 cavities +6 metamaterial unit cells) in the propagation direction. As shown in Fig. 4(a) and (b), we observed that the resonance modes are split into two and three distinct modes that are similar to the PC cavity structure for 2-cavity and 3-cavity systems, respectively. In PC cavity structures, when two isolated cavities are brought together, the localized photon modes should overlap. Due to this interaction, the doubly degenerate eigenmode splits into two distinct modes: symmetric and anti-symmetric. The measured
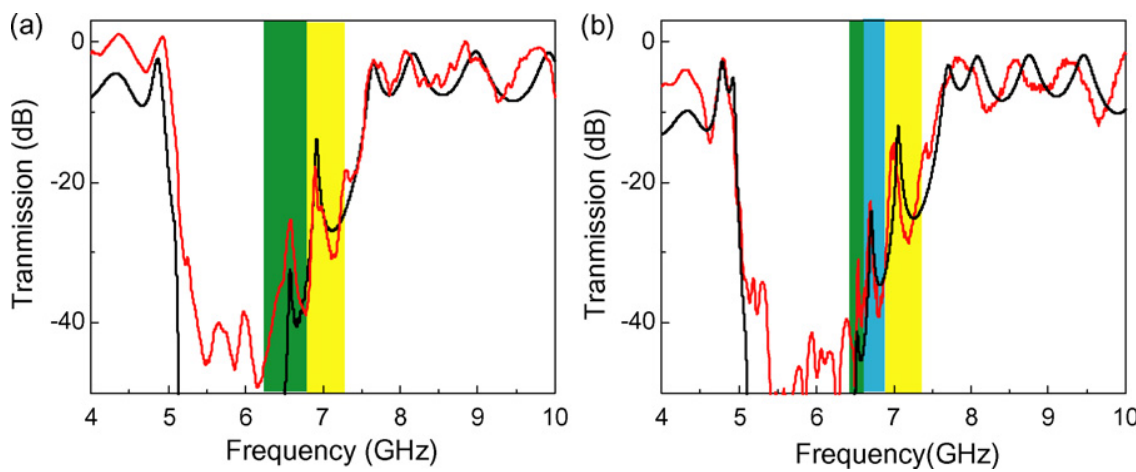

Fig. 4. Measured (red curves) and calculated (black curves) transmission spectra for (a) SRR-based 2-cavity and (b) 3-cavity systems. Due to the coupling between the strongly localized cavity modes, the single-cavity mode splits into two or three distinct modes depending on the number of coupled cavities. There is good agreement between the measured and calculated transmission spectra. (For interpretation of the references to color in this figure legend, the reader is referred to the web version of the article.) 


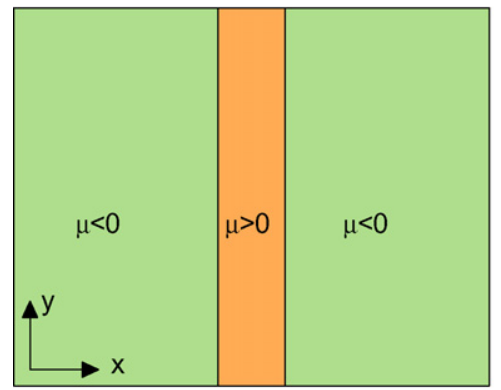

(a)

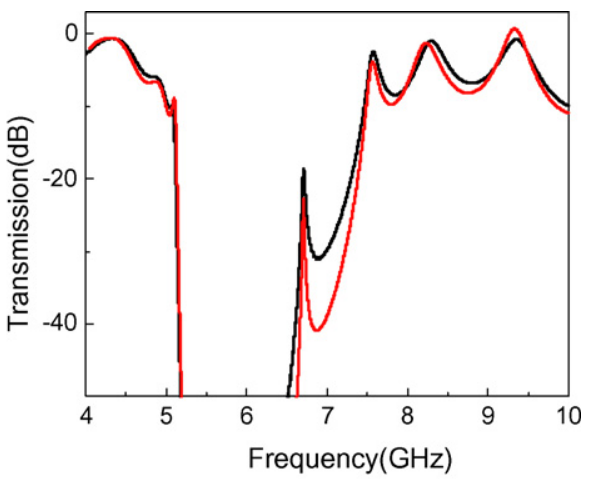

(b)

Fig. 5. (a) The SRR-based cavities can be treated as 1D FPRs with a subwavelength cavity region at the center. (b) The calculated transmission from the model of the SRR-based single cavity structure using the 1D transfer matrix method (red line) and CST Microwave Studio (black line) are in good agreement. (For interpretation of the references to color in this figure legend, the reader is referred to the web version of the article.)

values of the resonance frequencies for 2-cavity system are: $\omega_{1}=6.5 \mathrm{GHz}$ and $\omega_{2}=6.9 \mathrm{GHz}$. The resonance frequencies were measured as $\Gamma_{1}=6.4 \mathrm{GHz}, \quad \Gamma_{2}=$ $6.7 \mathrm{GHz}$, and $\Gamma_{3}=7.0 \mathrm{GHz}$ for the SRR-based 3-cavity system. The results are very similar to the PC coupled cavity systems. However, in taking advantage of the subwavelength dimension of the metamaterial system, it is possible to localize the fields into subwavelength regions using SRR-based cavity systems.

\section{1D Fabry-Perot resonator model}

The reflection of the SRR structure is very high in the negative $\mu(\varepsilon)$ frequency range and, therefore, the SRR structures on both sides of the cavity behave like frequency-specific mirrors [28,29]. Any propagating light that is trapped between them will bounce back and forth between these two mirrors. Since the mirrors localize light within a finite region, the modes are quantized into discrete frequencies, just as in FabryPerot resonances.

Therefore, it is possible to model these cavity structures as 1D FPR with the subwavelength uniform cavity region at the center (Fig. 5(a)). One of the important properties of the SRR system is to have a unit cell that is much smaller than the operating wavelength $(\lambda / 8)$. Therefore, the metamaterials can be treated as a homogeneous medium with an effective refractive index $[17,18]$.

By taking full advantage of this FPR model, we calculated the transmission from the SRR-based cavity structure using the $1 \mathrm{D}$ transfer matrix method. In the TMM calculations, we used effective $\varepsilon$ and $\mu$ that were determined by use of the retrieval procedure and Fresnel coefficients (reflection coefficient and transmission coefficient) for an interface when one of the two media is left-handed [30]. The calculated transmission using 1D TMM and CST Microwave Studio are in good agreement (Fig. 5(b)). Therefore, it is also in agreement with the measurements.

\section{Reduced photon lifetime}

A medium with a reduced group velocity, or in other words a medium with increased photon lifetime, offers a promising tool to increase the spontaneous emission rate and efficiency of nonlinear processes. Therefore, metamaterial-based cavities with a controllable group velocity can bring significant advantages for optoelectronic devices. The photon lifetime is defined as $\tau_{\mathrm{p}}=\partial \varphi /$ $\partial \omega$. Here, $\varphi$ is the net phase difference between the phase of the EM waves propagating inside the structure and the phase of the EM waves propagating

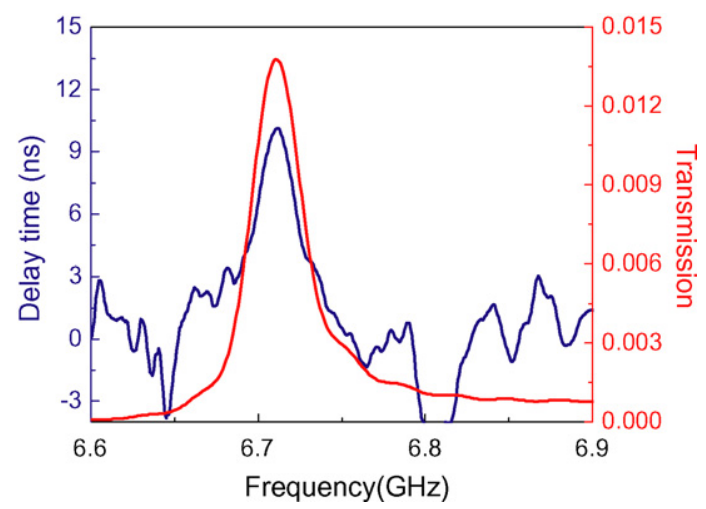

Fig. 6. The photon lifetime is increased at the cavity resonance of the SRR-based single cavity. Hence, the effective group velocity is reduced by a factor of 67 for an SRR cavity when compared to the electromagnetic waves propagating in free space. 
in free space for a total structure length. The photon lifetime corresponds to the propagation time of the EM waves inside the structure. The effective group velocity is inversely proportional to the photon lifetime and is defined as $L / \tau_{\mathrm{p}}$ where $L$ is the cavity length. The measured photon lifetime is $10 \mathrm{~ns}$ for an SRR cavity structure (Fig. 6). This also means that, at the cavity resonance, the effective group velocity is reduced by a factor of 67 for an SRR cavity when compared to the electromagnetic waves propagating in free space. The Q-factor and effective group velocity reduction factor are related through the localization of EM waves.

\section{Conclusion}

In conclusion, we showed that it is possible to obtain a cavity structure by the deformation of a unit cell of an SRR structure. We presented the Q-factor of the cavity resonance as 192 for the SRR-based single cavity. Moreover, it is possible to obtain a Q-factor as high as 3290 using loss free materials. The transmission properties of the SRR-based interacting 2-cavity and 3 -cavity systems were investigated. Due to the coupling between the strongly localized cavity modes, the singlecavity mode splits into two or three distinct modes, depending on the number of coupled cavities. Eventually, in taking full advantage of the effective medium theory, we modeled the SRR-based cavities as 1D FPRs with a subwavelength cavity at the center. The calculated transmission from the 1D FPR model using 1D TMM is in good agreement with the measured result. Finally, we observed that at the cavity resonance, the effective group velocity was reduced by a factor of 67 for an SRR-based single cavity compared to the electromagnetic waves propagating in free space.

This work is supported by the European Union under the projects EU-METAMORPHOSE, EU-PHOREMOST, EU-PHOME, and EU-ECONAM, and TUBITAK under the Project Numbers 105E066, 105A005, 106E198, 106A017, and 107A012. One of the authors (E.O.) also acknowledges partial support from the Turkish Academy of Sciences.

\section{References}

[1] J.B. Pendry, A.J. Holden, D.J. Robbins, W.J. Stewart, IEEE Trans. Microwave Theory Tech. 47 (1999) 2075.

[2] D.R. Smith, W.J. Padilla, D.C. Vier, S.C. Nemat-Nasser, S. Schultz, Phys. Rev. Lett. 84 (2000) 4184
[3] P. Gay-Balmaz, O.J.F. Martin, J. Appl. Phys. 92 (2002) 2929.

[4] R.A. Shelby, D.R. Smith, S.C. Nemat-Nasser, S. Schultz, Appl Phys. Lett. 78 (2001) 489.

[5] M. Bayindir, K. Aydin, P. Markos, C.M. Soukoulis, E. Ozbay, Appl. Phys. Lett. 81 (2002) 120.

[6] K. Li, S.J. McLean, R.B. Greegor, C.G. Parazzoli, M.H. Tanielian, Appl. Phys. Lett. 82 (2003) 2535.

[7] K. Aydin, K. Guven, M. Kafesaki, L. Zhang, C.M. Soukoulis, E. Ozbay, Opt. Lett. 29 (2004) 2623.

[8] K. Aydin, K. Guven, C.M. Soukoulis, E. Ozbay, Appl. Phys. Lett. 86 (2005) 124102.

[9] F. Martin, F. Falcone, J. Bonache, R. Marques, M. Sorolla, IEEE Microwave Wireless Comp. 13 (2003) 511.

[10] J. Martel, R. Marques, F. Falcone, J.D. Baena, F. Medina, F. Martin, M. Sorolla, IEEE Microwave Wireless Comp. 14 (2004) 210.

[11] K. Aydin, K. Guven, M. Kafesaki, C.M. Soukoulis, E. Ozbay, Opt. Exp. 12 (2004) 5896.

[12] T. Weiland, R. Schuhmann, R.B. Greegor, C.G. Parazzoli, A.M. Vetter, D.R. Smith, D.C. Vier, S. Schultz, J. Appl. Phys. 90 (2001) 5419.

[13] P. Markos, C.M. Soukoulis, Phys. Rev. E65 (2002) 036622.

[14] P. Markos, C.M. Soukoulis, Opt. Exp. 11 (2003) 649.

[15] R.W. Ziolkowski, IEEE Trans. Antennas Propag. 51 (2003) 1516.

[16] N. Katsarakis, T. Koschny, M. Kafesaki, E.N. Economou, C.M. Soukoulis, Appl. Phys. Lett. 84 (2004) 2943.

[17] T. Koschny, P. Markos, D.R. Smith, C.M. Soukoulis, Phys. Rev. E 68 (2003) 065602(R).

[18] T. Koschny, M. Kafesaki, E.N. Economou, C.M. Soukoulis, Phys. Rev. Lett. 93 (2004) 107402.

[19] Y.J. Hsu, Y.C. Huang, J.S. Lih, J.L. Chern, J. Appl. Phys. 96 (2004) 1979.

[20] R. Marques, F. Mesa, J. Martel, F. Medina, IEEE Trans. Antennas Propag. 51 (2003) 2572.

[21] J.D. Baena, R. Marques, F. Medina, J. Martel, Phys. Rev. B 69 (2004) 014402.

[22] B. Sauviac, C.R. Simovski, S.A. Tretyakov, Electromagnetics 24 (2004) 317.

[23] M. Shamonin, E. Shamonina, V. Kalinin, L. Solymar, J. Appl. Phys. 95 (2004) 3778.

[24] A.B. Movchan, S. Guenneau, Phys. Rev. B 70 (2004) 125116.

[25] T.J. Yen, W.J. Padilla, N. Fang, D.C. Vier, D.R. Smith, J.B. Pendry, D.N. Basov, X. Zhang, Science 303 (2004) 1494.

[26] S. Linden, C. Enkrich, M. Wegener, J. Zhou, T. Koschny, C.M. Soukoulis, Science 306 (2004) 1351.

[27] K. Guven, K. Aydin, E. Ozbay, Photonics Nanostruct. 3 (2005) 75.

[28] K. Aydin, E. Ozbay, IET Microwaves Antennas Propag. 1 (2007) 89

[29] E. Ozbay, Koray Aydin, E. Cubukcu, M. Bayindir, IEEE Trans. Antennas Propag. 51 (2003) 2592.

[30] C. Caloz, T. Itoh, Electromagnetic Metamaterials: Transmission Line Theory and Microwave Applications: The Engineering Approach, John Wiley \& Sons Inc., 2006 (chapter 2). 